\title{
Emotion in Artificial Intelligence and Artificial Life Research: Facing Problems
}

\author{
Jackeline Spinola de Freitas*, Ricardo R. Gudwin, João Queiroz ${ }^{* *}$ \\ Department of Computer Engineering and Industrial Automation \\ School of Electrical and Computer Engineering \\ State University of Campinas \\ PO Box 6101 - 13083-852 Campinas, SP - Brazil \\ \{jspinola, gudwin, queirozj\}@ dca.fee.unicamp.br
}

\begin{abstract}
Psychology and cognitive neuroscience researches are increasingly showing how emotion plays a crucial role in cognitive processes. Gradually, this knowledge is being used in Artificial Intelligent and Artificial Life areas in simulation and cognitive processes modeling. However, theoretical aspects of emotion to be employed in computational systems projects are scarcely discussed and very few comparisons are made between projects. Besides, we can notice that there are many open questions emotion-based projects might face to field development. This paper intends to discuss these problems and propose tentative directions to solve them.
\end{abstract}

\section{Introduction}

In the last decades, neuroscience and psychology research findings about emotion ([47], [20], [21], [22], [52], [32], [6], [59], [36]), are increasingly attracting the attention of many researchers in Computer Science and Artificial Intelligence (AI) areas (see [18], [57], [56], [34]).

These areas are especially interested on new scientific beliefs that emotions play an essential role in human cognitive processes and about its importance for problem solving competence and decision-making. Even if, since 1872 Darwin's [23] evolutionary theories indicated that emotions are evolved phenomena with important survival functions that have helped us solve certain challenges during our evolution, only recently emotion association with irrationality idea and non-logical behavior in human beings was reviewed ([48], [60], [11], [38], [3], [22], [40]).

AI and Artificial Life (Alife) areas, interested in cognitive processes modeling and simulation, clearly see that emotion is a crucial element to model perception, learning, decision process, memory, behavior and others functions they are interested in.

These facts were fundamental to produce an increasing number of theoretical and experimental projects both in $\mathrm{AI}$ and Alife and currently two computer science areas use emotion on their research.

Sibling area is Human-Computer Interaction (HCI) ([61], [27], [58], [10], [9], which focus on the interactions between man and machines and possible improvements on this relation. Researcher's intention is to development engineering tools to measure, model and respond to emotions with new sensors, algorithms and hardware devices. 'Affective Computing', a term coined by [58], is used to classify projects in this category and a successful commercial project example is AIBO Sony toy (http://www.sony.net/Products/aibo/). Face to advances already achieved $\mathrm{HCI}$ researchers are exploring emotion as a way to improve their implementations results and applications.

Second area involves emotion-based internal architecture systems ([69], [48], [51], [18], [7], [56], [34], [35]) with an attempt to also evolve it ([24], [62], [43]). Within this area, further categorization is possible and can be found, for example, in [15] and [72]. Researchers focuses are on computational architectures whose models are biologically inspired by emotion process studied by neuroscience with the intention of including emotions in machines processes control or to evolve an 'artificial emotion'. In general, emotion-based projects expect that including an emotion model into computational system they can improve machine performance in terms of decision-making competence, action selection, behavior control and autonomous and trustworthy system response.

\footnotetext{
* Supported by CNPq - Brazil.

** Supported by FAPESP - Brazil.
} 
Homogeneuosly, emotion-based computational systems still face many basic problems. Although we are particularly interested in second area, this paper intends to discuss subjects and problems related to both areas. Next section comments these problems but first gives some projects examples in each area. Section 3 emphasizes the lack of a framework adoption by projects and suggest one possible approach. Section 4 describes still open questions projects may not be facing and points out the lack of comparisons inter and intra projects. Last, section 5 is dedicated to final comments.

\section{Emotion-Based Computational Systems Problems}

For some years, experimental research using emotion-based agents is being developed. Examples of HumanComputer Interaction projects are: [61], [27], [58], [10], [16] and MIT Research Group ${ }^{1}$ projects such: Socially Intelligent characters, Embodied Conversational Agents and Story-Listening Systems.

Some discussed projects in AI context are: [12], [13], [14], [15], [48], [68], [69], [70], [18], [33], [34], [35], [41], [42], [7], [2], [62] and [31].

In Alife area it seems that very few projects are exclusively dedicated to emotion emergence. We could mention [43], which measure emotion as behavior modulation, i.e., considering particular modulations patterns representing the emotion emerged. Next, [37] in which different levels of an artificial hormone mechanism generate emotion. It is seemed as a consequence of a series of modifications on system resulting in emergent behavior. Likewise, [24] proposed a dynamic non-linear emotion model achieved through behavior changes from interactions across different levels of agent architecture but author affirms that "whether it will suffice to provide 'believable' emotional behavior is unclear at this time".

Even considering current state of the art projects, building emotion-based systems is far from being a straightforward job. Indeed, computational conceptions of emotion are as problematic and complex as computational understandings of life. Through mentioned projects and our viewpoint, we would say that problems are threefold.

First, (i) the lack of a well defined scientific framework to approach 'Artificial Emotion' (AE). It is a known constraint into AE research community and several attempts (e.g. [37], [69], [70], [71], [24], [68], [7], [50], [41], [42], [63]) have been published suggesting one. However, few of them (e.g. [12], [65], [4]) show advanced knowledge to follow, approaches that might be appropriately used to model emotions in autonomous agents. In spite of that, we hardly ever see references for previous frameworks in subsequent experimental projects as if they are always taking a new research way.

Besides problems of appropriate approaches or frameworks to model emotion, a close look at some projects provides a non-exhausted list of (ii) important questions projects might face to achieve trustworthy results. We even think that answering some of them is a must to emotion-based projects be taken seriously.

Last but not least these facts mainly contribute to a third noticeable problem: (iii) lack of comparisons between projects and also within same project, with comparative results from emotion and non-emotion-based experiments.

Before discussing these problems, we would mention that they may also apply to another category that could be defined as a combination of HCI and emotion-based internal architecture systems. However this field is still a distant goal.

\section{Emotion-Based Projects Frameworks}

Maybe we can interpret these faults as a tentative of achieving a surmountable idea as no project has proved being, until today, remarkable, prominent, distinguished. Or it can be seen as if available information about emotion phenomenon and its relation with other sub-systems to attain such qualities are not enough yet. Anyway, we believe, as [40] argue that "the development of computational models of emotion as a core research focus for artificial intelligence" can provide many advances of such systems.

Feasible framework suggestions are posed by [12], [15], [30] and [4], that it is necessary to pursue a functional view of the emotions presented in natural systems and we are going to concentrate on them.

In [30] we can see an extensive list of possible emotion functions such as change in autonomic and endocrine system, triggering motivated behaviors, communication, social bonding, improving survival, facilitating

\footnotetext{
${ }^{1}$ Massachusetts Institute of Technology. http://affect.media.mit.edu/projects.php
} 
memory storage and recall. But, although suggesting a functional view of emotions author list few functions of "emotions that have natural robotics counterparts" ([30]).

In [15], author proposes a functional view about the role of emotions in agent architecture and its implications for the design of emotional agents. She affirm that models of emotions that establish a link between emotion, motivation and behavior provided by a synthetic physiology structure can allow adequate conclusions as she previously achieved ([12]).

Furthermore, as [63] pointed out, it is worth asking whether emotions in artificial systems could have a potential role in action selection, adaptation, social regulation, sensory integration, alarm mechanisms, motivation, goal management, learning, attention focus, memory control, strategic processing and self model. But such complexity level certainly requires a wide project if we could say it would be able to manage that.

[4] propose that due to deepening understand of interactions between brain structure and neural mechanisms rooted in neuromodulation ${ }^{2}$ that underlie emotions in human and other animals ([19], [47], [20], [21]) it is possible to "abstract from biology a functional characterization of emotion"([4]). But authors alert that although tight interactions between amygdala and prefrontal cortex and its influence to generate emotion is already known, how this is done and how computational systems can take advantage of it remains an open question. Based on [44] analysis of motivation and emotion [4] propose, as a functional framework for emotion-based architectures, neuromodulatory systems dynamics to yield behavior emotional states even if they stress that emotions are "far more complex than a few brain structures" and interacting neuromodulatory systems.

Although complex, functional approach seems to be a possible framework to pursue more convincing emotion-based projects. As examples, but without questioning their plausibility, we can mention some projects that abstract animals' physical components, like hormonal levels, to represent emotion: [37], [33], [34], [2] and [73]. On the other hand, in the emotional control processes developed in [62], the author assumes "a general characteristic of emotion processes without making any claims about the biological plausibility of the employed states or mechanisms". It may be viewed as an initial approximation of concept that can help concretize psychological process theories ([40]). Indeed, some projects (e.g. [62]) observe evolved phenomenon and compares it with something that one could classify as emotions. As affirms [5], maybe it is a matter of a creative programmer who works metaphorically.

In fact, some projects deserve severe criticism about saying they include emotion, whether or not it's just the word they use without any approximate equivalence to emotion in natural systems. Admittedly, as [39] and [15] question, how much of observed emergent behavior is genuine and how much is conferred by an observer tendency to anthropomorphism is a difficult limit to establish.

\section{Some Questions for Emotion-Based Computational Systems}

As any new research area, we can assure that there are much more unanswered questions than problems solved. Positively, this fact can be viewed as an open opportunity for new proposals. But, to answer them, as [5] proposes, the effective tradition of foundational scientific research, to go back to first principles in order to grapple with an issue, should be pursue. Afterwards, the development of new computational implementations might help to solve or shed some light on most of them. Mentioned questions can be grouped in two types, related to (i) theoretical-conceptual problems or (ii) computational problems.

Scientific communities, as already cited, do not have a completely agreed definition for emotion, in spite of decades of attempts. Questions concerning the origins and functions of emotion, the relation between emotion and other affective processes (motivation, moods, attitudes, values, temperaments, etc) also seem to be difficult to get widely accepted responses. These facts may allow us to think that restricted understanding of mechanisms underlying emotion phenomenon can limit emotion-based systems progress. One possible solution to overcome that may be indeed to focus on the functions of emotions. Instead of thinking in "what emotions are", we should think in "what emotions are for" ([30]).

A list of important questions must include: how many and what emotions should be selected to be in an emotion-based system? Is there possible to have a feasible model that considers artificial emotions co-occurrence? Quantity and which emotions to select are two non-consensual questions between researchers. Four 'basic emotions' are considered in many systems: joy, sadness, anger and fear (e.g. [48], [34]). But according to [55] this number must very be bigger (15): happiness, sadness, anger, boredom, challenge, hope, fear, interest, contempt, disgust, frustration, surprise, pride, shame and guilt. [59] believes that eight emotions must be used, classified by him as primary emotions: joy, sadness, acceptance, anger, fear, disgust, anticipation and surprise. Contrar-

\footnotetext{
${ }^{2}$ See http://www.neuromodulation.com/
} 
ily, [57] argue that from theoretical point of view it is a fallacy to simply equate commonly described state of affairs with the existence of some fixed number of basic emotions and stress that Paul Ekman, the originator of the term 'basic emotions', has pinpointed the fact that he does no longer "allow for 'non-basic' emotions" ([26]). Given complex system control necessity, some recent projects (e.g. [62], [25]) seem to be inclined to select just one or two emotions. For us, it seems that best parsimonious suggestion is posed by [15]: "do not put more emotion in your system than what is required by the complexity of the system-environment interaction".

On the relation between emotion and other sub-systems: how to integrate it with other mechanisms, such as: sensory, learn, selection, reaction and communication? [56] want to know if the lack of integration between emotions and other systems (cognitive, language, memory, etc) impairs better global results. [15] affirms that for emotional mechanisms are able to interact simultaneously with several behavioral and cognitive subsystems like the physiological, the cognitive-evaluative and the communicative-expressive, they can provide an interesting solution to improve agents performance but author questions if such complexity is really necessary and currently possible. Including many interconnected subsystems, [50] proposes, 'The Emotion Machine', a sixlayered architecture of mind as a reliable model to solve that but, until the moment, it is theoretical and offers no easy implementation in a machine.

According to psychology ([54]) and neuroscience ([8], [20], [21], [22]) research emotions must be thought as processes that control cognition and action, that manage our mental models, frequently incomplete and incorrect. So, does the lack of emotions impair cognitive abilities in artificial autonomous agents? For [30] it is "clear that emotions have co-evolved with perceptual, cognitive and motor abilities" and "affect brain areas involved at all levels of functions, from low-level motor control to planning and high-level cognition". In this sense, comparisons between emotion and non-emotion-based projects may be helpful to provide information about emotion-cognition intertwinements.

Some questions that might be especially interesting for Alife projects can be associated ([13], [14], [15], [66], [62]) with emergent phenomena: can artificial emotion be an emergent property? if yes, how can design influence emergence of complex actions in emotion-based agents? [15] affirm that it is possible to let an agent evolve its own emotion and that it is a useful way to investigate the role emotions play in agent-environment situations of different levels of complexity. However, she points out that, to avoid possible problems, some functional equivalence between features of the agent and its environment must be preserve. In this context, problems are related to anthropomorphism tendency and also, since artificial system may be far from existing natural models it can be difficult to say "why and when emotional behavior arises" ([15]). [72] even question if it makes sense to speak of emotion in this case.

As [30] and [43] affirm that typical explanations for emotion function are based on flexibility of behavior response and [32] defines the core of an emotion as the readiness to act in a certain way, we can try to use behavior as a measurable phenomenon to emotion. Indeed, it is possible that, because of the lack of formal theories that describe a non-observable subjective emotion process ([34]) or intuitive parameters ([72]), many experiments ([37], [45], [43]) 'identify' emotion through observable resulting behavior.

We suspect that one of most discussed questions in AI is: Do emotion processes need to be related to an embodied 'entity'? In [17] philosophical view and [20], [21], [22] neuroscience background, body is essential for emotion process. But for [66] it is exactly the opposite when this question involves a computational apparatus. In between, [51] believes that an "emotion systems involved in feedback control of situated agents may serve to provide the grounding for embodied agents in the body/environment coupling".

Closely related to computational problems, we can identify other questions. [50] affirms that many of these problems, not solved yet, are related to a wrong way agents are programmed, a predefined algorithm instead of allowing agent to developed parts of its architectures during environment interactions.

Related to system architecture, some questions are: what kind of data structure and computational mechanisms should be used to both capture and represent the complexity of emotion processes? what emotion architectures models are better suited for agents performance comparison? [53] affirm that it is fundamental to overcome the challenges of identifying methods of encoding information that are suitable to produce incremental growth process. Thinking about obtain incontestable results we can ask what kind of experimental test allows to better explore emotion-based models. Particularly, we feel as if something is missing regarding computational tools to represent emotion phenomena and while we do not see new perspectives we must work hard to emotion abstraction to the extent that not to miss important brain structure interactions and not to be too complex that be prejudicial to computational representation.

As previously said, this section shows a non-exhausted list of problems (for more questions see, for example, [15]). And answering that is still a very difficult task, since it requires concepts that are not yet generally understood and theories that are not yet accepted or established ([15], [67]). Notable complexity of a system pro- 
ject that could answers cross-disciplinary questions, including necessary parameters to control so many factors implies that no such comprehensive project has been developed yet.

We truly believe that many of these questions are probably made at the beginning of research projects but, curiously, they have not been on discussion focus of publications in AI and Alife area.

Third mentioned problem seems to be the 'least difficult to solve': lack of comparisons between projects. Interaction among experiments could be useful to compare and discuss different architectures and, eventually, benefit projects course and generate more expressive results in shorter time. A systematic analysis of projects results is necessary to make research in artificial emotion better founded ([15]). Comparisons between emotional and non-emotional agent architecture within same experiment may also be a powerful way to validate conclusions.

\section{Final Comments}

Even if currently available knowledge about emotion has allowed that AI and Alife researchers propose models of emotion-based systems, an essential question to be answered is related to which extent supposed structural complexity involved in emotion phenomena can be abstracted and modeled. Indeed, the lack of appropriate frameworks for common reflection and of standards for a sound validation practice ([1]) are bottlenecks that need to be transposed.

As neuroscience findings increase, they might be more and more useful in the construction of emotion-based autonomous agent systems. Computational projects with particular focus will be able to extend their scope to include connected sub-systems. On the other hand, continuity of emotion-based computational experiments can be a way to have clues about unknown mind functions, a test-bed for theories of biological emotion ([4]).

The extent to which researches in AI and Alife will improve our understanding of mind phenomena and allow us to develop new robust and trustworthy artifacts will depend on the extent we will be able to answer remain open questions. Some hard questions require a broad and deep multidisciplinary background or such a research group that include, for example, psychologists, ethologists, neuroscientists, computer scientists, software engineers and philosophers. Even though it do not guarantee that it is possible to have a single model that responds the majority of questions. Attempts to answer these questions can also serve to show other limits emotion-based research might face, helping surpassing them.

Commonly made salutary critics and comparisons between projects can be a beneficial counterpart to experiments progress and development.

Positively, overcome this challenges can be an important step to field progress goes beyond engineering applications and towards a more scientific discipline ([1]).

\section{References}

1. AAAI press (2004). In Hudlicka, E. and Cañamero, D. (eds.) "Architectures for modeling emotion: cross-disciplinary foundations". 2004 AAAI Spring Symposium Technical Report. Available at: http://www.aaai.org/press/reports/symposia/spring/ss-04-02.html Accessed on 11/05/2004.

2. Almeida, L. B.; Silva, B. C. and Bazzan A. L. (2004) "Towards a physiological model of emotions: first steps". In Hudlicka, E. and Cañamero, D. (eds.) "Architectures for modeling emotion: cross-disciplinary foundations". 2004 AAAI Spring Symposium Technical Report. Available at: http://www.inf.ufrgs.br/ mas/emotions/ingles/PMEaaai04.pdf Accessed on 11/05/2004.

3. Anderson, Steven; Bechara, Antoine; Damásio, Hanna; Tranel, Daniel and Damasio, Antonio R. (1999) "Impairment of social and moral behavior related to early damage in human prefrontal cortex". Nature Neuroscience v. $2 \mathrm{n}^{\circ} 11$, November, 1999 pp. 1032-1037.

4. Arbib, M. A. and Fellous, J-M. (2004) "Emotions: from brain to robot”. Trends in Cognitive Sciences. V. 8 n 12 , pp. 554-561.

5. Arzi-Gonczarowski, Zippora. (2002) “AI Emotions: Will One Know Them When One Sees Them?” Agent Construction and Emotions at 16th European Meeting on Cybernetics and Systems Research. Vienna, Austria, April, 2002. Available at: http://users.actcom.co.il/typographics/zippie/zprz_emcsr02.pdf Accessed on 11/05/2004.

6. Barkow, J. H.; Cosmides, L. and Tooby, J. (1992) (eds.) "The Adapted Mind: Evolutionary Psychology and the Generation of Culture". New York, NY: Oxford University Press, 1992.

7. Bazzan, A. L. C. and Bordini, R. (2001) "A framework for the simulation of agent with emotions: report on experiments with the iterated prisoner`s dilemma" In: The 5th Int. Conference on Autonomous Agents, Montreal, New York: ACM. 2001. pp. 292-299. 
8. Bechara, Antoine; Damásio, Hanna and Damásio, Antonio R (2000) "Emotion, Decision Making and the Orbitofrontal Cortex". Cerebral Cortex, Oxford University Press V. 10, n 3, pp. 295-307.

9. Brave, Scott and Nass, Clifford. (2003) "Emotion in human-computer interaction". The human-computer interaction handbook: fundamentals, evolving technologies and emerging applications. Lawrence Erlbaum Associates, Inc: Mahwah, NJ, USA, pp. 81-96.

10. Breazeal, Cynthia. (2003) "Emotion and sociable humanoid robots". International Journal of Human Computer Studies, 2003, pp. 119-155.

11. Bryson, Joanna and Flack, Jessica (2001) "Emotions and Action Selection in an Artificial Life Model of Social Behavior in Non-Human Primates" Available at: http://citeseer.ist.psu.edu/491375.html Accessed on 10/12/2004.

12. Cañamero, D. (1997) "Modeling Motivations and Emotions as a Basis for Intelligent Behavior". In: First Conference on Autonomous Agents, 2001, Marina Del Rey, California: ACM. 1997. pp. 148-155.

13. Cañamero, D. (1998) "Issues in the Design of Emotional Agents". In Proceedings of the 1998 AAAI Fall Symposium. Emotional and Intelligent: The Tangled Knot of Cognition. Menlo Park, CA: AAAI Press, pp. 49-54.

14. Cañamero, D. (2000) “Designing Emotions for Activity Selection”. Dept. of Computer Science - Technical Report DAIMI PB 545, University of Aarhus, Denmark. Available at: http://www.daimi.au.dk/PB/545/PB-545.pdf Accessed on $06 / 14 / 2004$

15. Cañamero, D. (2001) "Emotions and adaptation in autonomous agents: a design perspective". Cybernetics and systems: international journal. V. 32, 2001,pp. 507-529.

16. Cassell, Justine. (2004) Home Page. Available at: http://www.soc.northwestern.edu/justine/ Accessed on 06/12/2004.

17. Clark, A.; Slomam, Aaron; Zeman, Adam and Oberlander, Jon (2004) "Can computers have emotions?" The debate panel - position statements, November, 2004. Available at: http://www.inf.ed.ac.uk/events/hotseat/panel_statements.html Accessed on 01/12/2005.

18. Custódio, L.; Ventura. R. and Pinto-Ferreira C. (1999) “Artificial emotions and emotion-based control systems". Proceedings of 7th IEEE International Conference on Emerging Technologies and Factory Automation. V. 2. pp. 1415-1420, 1999.

19. Dalgleish, T. (2004)“The emotional brain”. Nature Reviews Neuroscience, 5, pp. 582-589.

20. Damásio, Antonio. (1994). "Descartes'error: emotion, reason and the human brain". New York, NY: Avon books.

21. Damasio, Antonio R.; Grabowski, T.; Bechara, A.; Damasio, H.; Ponto, L. L.; Parvizi, J. and Hichwa, R. D. (2000) "Subcortical and cortical brain activity during the feeling of self-generated emotions" Nature Neuroscience v. $3 \mathrm{n}^{\circ} 10$, October, 2000 pp. 1049-1056.

22. Damásio, Antonio R. (2001) "Emotion and the Human Brain". Annals of the New York Academy of Sciences v. 935, pp. 101-106.

23. Darwin, Charles. (1872). "The expression of the emotions in man and animals". Available at: http://www.darwinliterature.com/the_expression_of_the_emotions_in_man_and_animals/index.html. Accessed on 02/20/2004.

24. Davis, D. N. (2000) "Modelling emotion in computational agents." Available at: http://www2.dcs.hull.ac.uk/NEAT/dnd/papers/ecai2m.pdf Accessed on 02/20/2004.

25. Delgado-Mata, Carlos and Aylett, Ruth S. (2004) "Emotion and Action Selection: Regulating the Collective Behaviour of Agents in Virtual Environments". Third International Joint Conference on Autonomous Agents and Multiagent Systems. V. 3, New York City, USA, July, 2004, pp. 1304-1305.

26. Ekman, Paul. (1999) "Basic Emotions”. In Dalgleish, T. and Power, T. (eds.) The Handbook of Cognition and Emotion. Sussex, UK: John Wiley \& Sons, Ltd. 1999, pp. 45-60.

27. Elliott, Clark (1994) “The Affective Reasoning Project”. Available at: http://condor.depaul.edu/ elliott/arback.html Accessed on 02/20/2004.

28. Evans, Dylan. (2005) "Emotional robotics". Available at: http://www.dylan.org.uk/emobot.html. Accessed on $03 / 20 / 2005$.

29. Fellous, J-M. (1999) Neuromodulatory basis of emotion. The Neuroscientist, v. 5, pp. 283-294.

30. __ (2004) "From Human Emotions to Robot Emotions". In Hudlicka, E. and Cañamero, D. (eds.) "Architectures for Modeling Emotions: Cross-Disciplinary Foundations.” AAAI Spring Symposium. Menlo Park, CA. pp. 37-47.

31. Franklin, Stan. (2005) "How minds work - IDA and her architecture". Available at: http://www.cs.memphis.edu/classes/unhp4453/presentations/idaandherarchitecture.ppt Accessed on 03/20/2005.

32. Frijda, N. H. (1993). "The place of appraisal in emotion". In cognition and emotion. V. 7 pp. 357-387.

33. Gadanho, Sandra. (1999) "Reinforcement learning in autonomous robots: an empirical investigation of the role of emotions". Ph.D. Thesis. University of Edinburgh, 1999. Available at: http://omni.isr.ist.utl.pt/ sandra/papers/thesis.pdf Accessed on 03/22/2004.

34. Gadanho, S. and Hallam, J. (2001a) "Emotion-triggered learning in autonomous robot control". Cybernetics and Systems: an international journal. V. 32, July, 2001, pp.531-559.

35. Gadanho, S. and Hallam, J. (2001b) "Robot learning driven by emotions". Adaptive Behavior, 9 (1), 2001.

36. Ghiselin, Michael T. (1973). "Darwin and Evolutionary" Psychology. Science 179: 964-968.

37. Gomi, T. and Ulvr, J. (1993) "Artificial Emotions as Emergent Phenomena". In Proceedings of IEEE Workshop on Robot and Human Communication, Tokyo, Japan, November, 1993.

38. Goleman, D. (1995). "Emotional intelligence". New York: Bantam, 1995.

39. Grand, Stephen; Cliff, D. and Malhotra, Anil. "Creatures: Artificial Life Autonomous Software Agents for Home Entertainment” In: First Conference on Autonomous Agents, 2001, Marina Del Rey, California: ACM. 1997, pp. 22-29. 
40. Gratch, Jonathan and Marsella, Stacy. (2004) "A domain independent framework for modeling emotion," Journal of Cognitive Systems Research, v. 5, pp. 269-306, 2004.

41. Henninger Amy. E.; Jones, Randolph. M., and Chown, Eric. (2001). “A Symbolic-Connectionist Framework for Representing Emotions in Computer Generated Forces”. Proceedings of the 2001 Interservice/Industry Training, Simulation, and Education Conference. Orlando, FL.

42. _ (2003) "Behaviors that Emerge from Emotion and Cognition: Implementation and Evaluation of a SymbolicConnectionist Architecture”. AAMAS’03, Melbourne, Australia, July, 2003.

43. Kato, Tomoko and Arita, Takaya. (2005) "Evolutionary Simulations based on a Robotic Approach to Emotion". The 10th International Symposium on Artificial Life and Robotics. Oita, Japan, February, 2005.

44. Kelley, A. E. (2004) "Neurochemical networks encoding emotion and motivation: an evolutionary perspective". In Who

Needs Emotions? The Brain Meets the Robot. Fellous, J-M. and Arbib, M. A (eds). Oxford University Press, 2004

45. Kitamura, T. (1998) "An Architecture of Behavior Selection Grounding Emotions." 5th International Conference of the Society for Adaptive Behavior (SAB'98). University of Zurich, Switzerland, August, 1998.

46. Langton, Christopher G. (1995) (ed.) “Artificial life: an overview”. Cambridge, Mass. and London, England: a Bradford book, the MIT Press, 1995.

47. Ledoux, Joseph. (1996) "The emotional brain: the mysterious underpinnings of emotional life”. New York, NY: Touchstone, 1996.

48. McCauley, T. L. and Franklin, S. (1998) "An architecture for emotion”. In Proceedings of the 1998 AAAI Fall Symposium. Emotional and Intelligent: The Tangled Knot of Cognition. Menlo Park, CA: AAAI Press, pp.122-127.

49. Minsky, Marvin. (1986) "The society of mind". New York: Simon and Schuster, 1986.

50. _ (2002). "The emotion machine". Available at: http://web.media.mit.edu/ minsky/e1/eb1.html Accessed on $01 / 06 / 2005$.

51. Nehaniv, C. (1998) "The First, Second, and Third Person Emotions: Grounding Adaptation in a Biological and Social World." 5th International Conference of the Society for Adaptive Behavior (SAB'98). University of Zurich, Switzerland, August, 1998.

52. Nesse, Randolph M. (1994) "Computer emotions and mental software”. Social neuroscience bulletin vol. 7, $\mathrm{n}^{\circ}$ 2, spring 1994, pp. 36-37.

53. Nolfi, Stefano and Floreano, Dario. (2002). "Synthesis of autonomous robots through artificial evolution". Trends in cognitive sciences 1, pp. 31-37.

54. Oatley, Keith (1999) In: Wilson, R. A.; Keil, F. C. (ed). The Massachusetts Institute of Technology - Encyclopedia of the Cognitive Sciences. Cambridge, Massachusetts e London, England: A Bradford book, The MIT Press, 1999, pp. 273275.

55. Ortony, Andrew; Clore, Gerald L. and Collins, Allan. (1988) "The cognitive structure of emotions". New York, NY: Cambridge university press, 1988.

56. Petta, Paolo and Cañamero, D. (2001) (eds.) "Grounding emotions in adaptive systems: volume II." Cybernetics and systems: an international journal. V. 32, 2001, pp. 581-583.

57. Petta, Paolo and Trappl, Robert (2001) "Emotions and agents”. Multi-agents systems and applications. Springer-Verlag: New York, NY, USA. pp. 301 - 316.

58. Picard, Rosalind. (1997) “Affective Computing”. Cambridge, Massachusetts: MIT Press, 1997.

59. Plutchik, R. (1980). "Emotion: a psycho evolutionary synthesis”. New York, NY: Harper \& Row.

60. Ray, P., Toleman, M. and Lukose, D. (2001). "Could emotions be the key to real artificial intelligence?" Available at: http://www.sci.usq.edu.au/research/workingpapers/sc-mc-0009.ps Accessed on 08/09/2004.

61. Reilly, W. S. and Bates, J. (1992) "Building Emotional Agents". Technical Report CMU-CS-92-143, School of Computer Science, Carnegie Mellon University, Pittsburgh, PA, May 1992. Available at: http://www2.cs.cmu.edu/afs/cs.cmu.edu/project/oz/web/papers/CMU-CS-92-143.ps Accessed on 09/11/2004.

62. Scheutz, Matthias. (2004a) "Useful roles of emotions in artificial agents: a case study from artificial life". In Proceedings of AAAI 2004. AAAI press, pp. 42-48. Available at: http://www.nd.edu/ airolab/publications/aaai104scheutzm.pdf Accessed on 11/12/2004.

63. _. (2004b) "An Artificial Life Approach to the Study of Basic Emotions". In Proceedings of CogSci 2004. Available at: http://www.nd.edu/ mscheutz/publications/cogsci04.pdf Accessed on 01/06/2005.

64. Sloman Aaron and Croucher. M. (1981) "Why robots will have emotions". In Proceeding IJCAI, June, 1981. Available at: http://www.cs.bham.ac.uk/research/cogaff/aaron.sloman_why_robot_emotions.pdf Accessed on 11/12/2004.

65. Sloman, A. (2002) "How many separately evolved emotional beasties live within us?" In Trappl, R., Petta, P. and Payr, S (eds.) Emotions in Humans and Artifacts. The MIT Press, pp. 35-114

66. Sloman, Aaron; Chrisley Ron and Scheutz, Matthias. (2003) "Who needs emotions? The brain meets the machine." In Arbib, M. and Fellous, J. (eds.) Oxford, New York: Oxford University Press. Available at: http://www.cs.bham.ac.uk/research/cogaff/sloman-chrisley-scheutz-emotions.pdf Accessed on 11/12/2004.

67. Sloman, Aaron. (2004) "What are emotion theories about?" In Hudlicka, Eva and Cañamero, D. (eds.) Architectures for modeling emotion: cross-disciplinary foundations. AAAI Spring Symposium Technical Report. pp. 128-134, 2004. Available at: http://www.cs.bham.ac.uk/research/cogaff/sloman-aaai04-emotions.pdf Accessed on 11/05/2004.

68. Staller, Alexander and Petta, Paolo. (1998) "Towards a Tractable Appraisal-Based Architecture for Situated Cognizers”. In Numaoka C., Cañamero D., Petta P. (eds.): Grounding emotions in adaptive systems, sab'98 (5th international conference of the society for adaptive behavior) workshop notes, Zurich, Switzerland. August 21, 1998. 
69. Velásquez, Juan. (1998a) “Modeling emotion-based decision-making”. In Cañanero, D. (ed.) Proceedings of the 1998 AAAI Fall Symposium. Emotional and Intelligent: the Tangled Knot of Cognition, pages 164-169. Available at: http://www.ai.mit.edu/people/jvelas/papers/velasquez-fs98.ps Accessed on 09/05/2004.

70. __ (1998b). "When Robots Weep: Emotional Memories and Decision-Making." Proceedings of the Fifteenth National Conference on Artificial Intelligence. Madison, WI, 1998, pp. 70-75.

71. __ (1998c) "A computational framework for emotion-based control". In Workshop on Grounding Emotions in Adaptive Systems, Conference on Simulation of Adaptive Behavior, 1998.

72. Wehrle, T. (1998). "Motivations behind modeling emotional agents: whose emotion does your robot have?" In Numaoka C., Cañamero D., Petta P. (eds.): Grounding emotions in adaptive systems, SAB'98 (5th international conference of the society for adaptive behavior) workshop notes, Zurich, Switzerland. August 21, 1998.

73. Wilson, Ian. (2004) "Simulating Artificial Emotion and Personality". In Hudlicka, E. and Cañamero, D. (eds.) Architectures for modeling emotion: cross-disciplinary foundations. AAAI Spring Symposium Technical Report. pp. 150-151, 2004. Available at: http://www.neon.ai/docs/SS204WilsonI.pdf Accessed on 09/05/2004. 